\title{
almenara
}

\section{La Escuela de Chicago tiene género: la criminóloga Frances Alice Kellor (1873 - 1952).}

\author{
Jordi Ortiz García ${ }^{1}$ \\ Santiago Cambero Rivero ${ }^{2}$
}

ORCID: http://orcid.org/0000-0002-0837-9372

Recibido el 5 agosto 2020

Aceptado el 1 de diciembre de 2020

\section{Resumen}

El presente trabajo pretende visibilizar la carrera profesional de Frances Alice Kellor, abogada y criminóloga, que desarrolló parte de su labor investigadora en la Escuela de Chicago, donde abordó investigaciones sobre la inmigración, las prisiones o la delincuencia femenina a principios del siglo XX. Frances Kellor realizó numerosas contribuciones sociológicas y criminológicas de gran interés científico, y desarrolló una brillante carrera pública en defensa de los derechos de las personas inmigrantes y de las mujeres. Este artículo de investigación analiza su vida, obras y carrera académica y profesional, que desafortunadamente ha sido invisibilizada, al igual que otras figuras femeninas contemporáneas. Al mismo tiempo, este artículo pretende iniciar una reflexión sobre la ausencia en los manuales de Criminología de tantas mujeres que contribuyeron al desarrollo de esta disciplina a lo largo de la historia.

Palabras Claves: Género; Feminismo; Escuela de Chicago; Prisión; Inmigración.

\begin{abstract}
The present work aims to make visible the professional career of Frances Alice Kellor, lawyer and criminologist, who developed part of her research work at the School of Chicago, where she studied on the immigration, the prisons and the female delinquency at the beginning of the 20th century. Frances Kellor made numerous sociological and criminological contributions of great scientific interest, and developed a brilliant public career in defense of the rights of immigrants and women. This research article analyzes her life, works, and academic and professional career, which unfortunately has been made invisible, as well as another contemporary prominent female. At the same time, this article intends to begin a reflection on the absence in the manuals of Criminology of women who contributed to the development of this discipline throughout history.
\end{abstract}

Keywords: Gender; Feminism; Chicago School; Prison; Immigration.

\footnotetext{
${ }^{1}$ Doctor en Derecho y Ciencias Sociales. Profesor de la Universidad de Extremadura.

${ }^{2}$ Doctor en Sociología. Profesor de la Universidad de Extremadura.
} 


\section{INTRODUCCIÓN}

“La revisión del espacio público, del poder y, a su vez, de los imaginarios colectivos no será posible si no nos tomamos en serio la igualdad de género en ámbitos como la cultura o la ciencia. Necesitamos construir conocimientos, saberes y relatos desde la incorporación de las mujeres como sujetos pensantes y creadores, rompiendo con la exclusividad de la mirada masculina y, por supuesto, haciendo visibles modelos de hombres que ejemplifiquen otras subjetividades”.

Octavio Salazar, $2018^{3}$

La historia es fundamental para la evolución de los pueblos y dejar fuera a la mitad de ella, simplemente por el hecho de ser mujeres, es sin duda un paso atrás. Este ha sido el sesgo de las ciencias hasta la edad contemporánea, y por supuesto, el hecho social de la segregación por género en distintos ámbitos de nuestras sociedades. El progreso debe ser participado por la población en general, al margen de sus condiciones personales, pues de lo contrario, los avances no se materializan en las estructuras sociales, políticas o económicas de la civilización occidental.

Sin entrar en detalle sobre la cuestión de género y las desigualdades sociales, esta investigación focaliza en una disciplina relativamente joven si la comparamos con otras consolidadas en el mundo científico, como sería la Criminología. Una ciencia humana e interdisciplinaria, cuyo origen histórico se remonta a finales del siglo XIX en Europa, y más concretamente en Italia con nombres propios que se citaran a continuación.

España como estudio de caso, al igual que otros países, ha sido una clara muestra de ese olvido e invisibilidad de brillantes mujeres que contribuyeron a la construcción de esta disciplina que estudia el delito, las víctimas y los criminales. A modo de ejemplo... modo de ejemplo, los manuales de Criminología y su historia en nuestro país, están marcados por el estudio de investigadores como: Lombrosso; Ferri; Beccaria, Sutherland, Becker; Merton; Sampson; Cohen; Bandura; Clarke; Skogan o Garland, pero también propios como: Salillas; Giner de los Ríos; Bernaldo de Quirós, Alfonso Serrano; Juan José Medina; Santiago Redondo o Vicente Garrido ${ }^{4}$. Importantes científicos que han contribuido y contribuyen de manera brillante a esta disciplina, pero que evidencian la falta de investigadoras y científicas en este campo, al menos de forma tan significativa como los anteriormente citados.

Otro aspecto importante, se refleja en la ausencia de estudios de la delincuencia desde una perspectiva de género. Las investigaciones se han centrado en el análisis de la variable género, y fundamentalmente en la distinción del hombre y la mujer como protagonistas del hecho delictivo, ya sea autor o víctima. Sólo recientemente y de forma un tanto ambigua, encontramos en nuestros manuales de Criminología, algún apartado sobre el papel de mujeres investigadoras en la Criminología 5 .

\footnotetext{
${ }^{3}$ Salazar Benítez, O. (2018). El hombre que no deberíamos ser (1ª Edición) Barcelona. Editorial Planeta.
}

\footnotetext{
${ }^{4}$ Serrano Gómez, A. (2007). Historia de la Criminología en España. Madrid. Dykinson.
}

${ }^{5}$ Serrano Tárraga, Ma D. (2014). El Rol de la Criminología para la Seguridad en la Sociedad Contemporánea. Madrid. Dykinson 233-234; Torres Díaz. M.C. (2018). Dret i Criminología: Guies per una docència universitaria amb perspectiva de génere. Xarxa Vives d'universitat. Disponible en: http://www.vives.org/serveis/guies-per-a-una-docencia-universitaria-ambperspectiva-de-genere/dret-i-criminologia/ 
En tercer lugar, y pese a que las participaciones de investigadoras en congresos científicos de la disciplina son numerosas y heterogéneas, como hemos podido observar en años anteriores en congresos de criminología celebrados en las ciudades de Sevilla y Oviedo ${ }^{6}$, dos eventos académicos con enorme éxito de participación, el número de mujeres (ponentes; moderadoras; conferencias inaugurales o clausura) ha sido mayor que el de hombres, pero continuamos encontrándonos en los programas de los congresos el término criminólogo en singular, para dirigirnos al profesional o experto en Criminología, obviando la forma femenina: criminóloga ${ }^{7}$.

En el ámbito profesional de la Criminología en España, también encontramos una brecha importante de género. Una prueba evidente es que los actuales Decanos o Presidentes de los Colegios Profesionales de Criminología sean hombres, demostrando así la escasa o nula presencia femenina en tales organizaciones corporativas, como sucede en otros órdenes de nuestra sociedad.

Estos datos son sólo una pequeña muestra de lo que indicábamos en los primeros párrafos de este artículo sobre la invisibilización de las mujeres en la Criminología. Con toda seguridad quedarían otros indicadores que se podría analizar ${ }^{8}$. A modo de ejemplo, y en la misma línea que estamos trabajando ¿Cuál es el número de doctoras que tenemos en Criminología en nuestro país frente al número de hombres?

En definitiva, una serie de indicadores que reflejan la posición relegada y degradada que ocupan generalmente las mujeres con respecto a los hombres, en el ámbito científico, académico y profesional de la Criminología. Frances Kellor, también fue postergada a pesar de sus logros como investigadora y reformadora social, dado los valores y las actitudes predominantemente machistas, a principios del siglo pasado en los Estados Unidos de América. Por consiguiente, tener la oportunidad de profundizar en la vida de esta mujer y sus orígenes científicos (Escuela de Chicago y Centro Hull - House) supone captar una parte mínima de la historia de la Criminología. Pero, sobre todo, visibilizar la labor científica que han hecho tantas mujeres a lo largo de la historia, en un momento significativo de la historia de la Criminología, como fue la Escuela de Chicago en Estados Unidos.

\section{CRIMINOLOGÍA FEMINISTA}

Antes de profundizar en la Escuela de Chicago, como impulsor de la Sociología de la desviación y la delincuencia, se podría confirmar que la Criminología parte de un sesgo machista y de clase baja a lo largo de su historia científica. De ahí, que surgiera la Criminología feminista en los años setenta del siglo pasado

${ }^{6}$ Congreso Andaluz de Criminología. Disponible en: https://cacriminologia.wordpress.com/\#post-96 [Última consulta el 15/04/2018]. XII Congreso de Criminología. Disponible en: https://criminologia.net/congresos/proximo-congreso/ [Última consulta el 20 de mayo de 2018]. En el Congreso Andaluz de Criminología en su programa el término criminólogo en varias ocasiones: MESA DE DEBATE (II). El criminólogo al servicio de la valoración pericial de riesgo delictivo; MESA DE DEBATE (III). El criminólogo al servicio de la atención a las víctimas de delito; El criminólogo y la justicia restaurativa en prisión; MESA DE DEBATE (IV). El criminólogo en el ámbito de la empresa privada. En el Congreso Nacional de Criminología celebrado en Oviedo podemos encontrar en el panel G4: La gestión del fraude como salida profesional del criminólogo. Disponible en: https://seicdifusion.files.wordpress.com/2018/06/programa-cecrim-2018-22_06.pdf. [última consulta 25/10/2020].

${ }^{7}$ A este respecto, existe a día de hoy un debate sobre el lenguaje inclusivo, teniendo en cuenta que la RAE acepta el masculino genérico. Desafortunamente, hoy continuamos observando cómo se llevan a cabo actividades académicas organizadas por instituciones de carácter criminológico, donde se recoge el término criminólogo, obviando el término criminóloga. A modo de ejemplo, podemos ver como el Colegio de Criminólogos de Madrid presentó el I Congreso de Criminología prospectiva, donde visibilizará la figura del criminólogo para lograr la presencia real en la función pública y privada. Disponible en: https://colegiocriminologosmadrid.es/congreso_criminologia_madrid_cpcm/. [última consulta 10/11/2020].

${ }^{8}$ Cabe recordar, que la Agenda 2030 para el desarrollo sostenible de Naciones Unidas y sus objetivos 5 y 11, muestran la importancia de abordar la igualdad de género para lograr un mejor desarrollo inclusivo y sostenible. Sobre este tema, puede verse: https://www.unwomen.org/es/news/in-focus/women-and-the-sdgs/sdg-5-gender-equality. [última consulta 05/11/2019]. 
para romper con las pautas de la Criminología clásica, siendo doblemente crítica con esta misma disciplina, ya que se desconocía a la mujer como delincuente y víctima, al mismo tiempo que se estudiaba de forma distorsionada la presencia femenina por el conjunto de estereotipos sexuales o psicológicos.

Los primeros estudios criminológicos pretendieron explicar qué caracteriza a las mujeres delincuentes partiendo de las características biológicas y psicológicas atribuidas como propias al sexo femenino. En resumen, las criminales féminas eran más masculinas que femeninas, llegando a pensar y comportarse como hombres. Por lo tanto, la delincuencia sólo podía relacionarse con rasgos propiamente masculinos, obviando la perspectiva de género. De modo, que las mujeres delincuentes se calificaban como personas anormales, degeneradas moralmente -aunque los hombres acudieran a la prostitución-, pasivas psicológicamente por su dependencia a los varones, y que actuaban fría y calculadamente por instintos básicos. En definitiva, eran consideradas como “monstruos”, definidas así por Lombroso y Ferrero.

En definitiva, la mujer ha resultado invisible para el desarrollo teórico de la Criminología, y cuando aparecía se le consideraba inferior, irracional, compulsiva y movida por impulsos primarios de tipo sexual y psicológico. La Criminología clásica basó sus estudios de delincuencia femenina en el heteropatriarcado y en los roles y estereotipos de género. Generalmente, el sesgo machista criminológico se observa en la proporción mayoritaria de estudios sobre la prostitución más que en la violación, y esta última, a veces inducida por las propias víctimas.

Por consiguiente, el surgimiento de la Criminología feminista fue una respuesta de reacción científicasocial, que pone el énfasis en las diferencias de género, denunciando el sesgo machista en los años sesenta en el desarrollo de la Criminología. Uno de sus objetivos más relevantes es visibilizar la delincuencia femenina como una delincuencia distinta a la masculina y donde no pueden extenderse en su totalidad las teorías tradicionales, que han sido creadas a partir de investigaciones y estudios en hombres. Así, la perspectiva de género se introduce en las propuestas feministas para entender el delito de y en la mujer, además de analizar el papel del género en los procesos de control social y desviación.

La cuestión de fondo seria la socialización diferenciada ${ }^{9}$ como proceso de adquisición de la identidad de género, que implica estilos cognitivos, conductuales y actitudinales, así como códigos morales diferentes según el género de la persona; es decir, la creación de normas estereotipadas asignadas a la conducta de cada persona en referencia a su género. Los resultados de la socialización diferencial por género han condicionado la evolución teórica de la Criminología, principalmente producida por hombres que ocultaban el rol de la mujer en la sociedad en general, bien masculinizando a las mujeres "malvadas" o bien explicando el carácter sexual de su desviación.

La Criminología feminista visibiliza a la mujer mediante investigaciones sobre mujeres, hecha por mujeres y para mujeres, que pretende entender el delito desde las diferencias de género, con metodología no sexistas y que contribuyan al empoderamiento femenino desde hace varias décadas ${ }^{10}$.

\section{LA ESCUELA DE CHICAGO}

Al margen de la reacción de la Criminología feminista comentada con anterioridad, hay pioneras en el ámbito de la Criminología, como es la figura sobre la que gira este artículo, Frances Alice Kellor. Una de las primeras fuentes de inspiración en la vida de esta investigadora fue su paso por la Universidad de Chicago, donde nace la denominada Escuela de Chicago, que ha desempeñado un papel principal en la historia más

\footnotetext{
${ }^{9}$ Walker, S. y Barton, L. 1983. Gender, class and education. Nueva York: The Falmer Press.
}

${ }^{10}$ Adler, F. (1975). Sister in Crime. The Rise of the new female criminal. New York. Mc - Graw-Hill. Y Simon, R.J. (1975). Women and Crime. Lexington, M.A: D.C. Health. 
reciente de la Sociología y la Criminología ${ }^{11}$. La Escuela de Chicago es conocida por las numerosas investigaciones, trabajos científicos y teorías elaboradas en el Departamento de Sociología de la Universidad de Chicago entre finales del siglo XIX y principios del siglo $\mathrm{XX}^{12}$.

La Escuela de Chicago enfatizó el pragmatismo, pues según sus representantes, el conocimiento debe estar integrado con la acción. La función de la Sociología es explorar esas experiencias y estudiar procesos sociales, tal y como son experimentados, de modo que estos sociólogos están interesados en el proceso de urbanización y la expansión en las ciudades. En esos contextos urbanos donde observan la delincuencia, como desviación concentrada en determinadas áreas de desorganización y desorden social con debilitamiento de los controles sociales (familia, vecindario, moralidad...), donde actúan bandas que luchan por defender un territorio o imponer sus normas de supremacía y respeto. Paradójicamente, la delincuencia es un esfuerzo local para restaurar el orden y las oportunidades ante un vacío de organización social.

La Escuela de Chicago, desde una perspectiva criminológica, analizó el fenómeno criminal en la ciudad de Chicago ${ }^{13}$. Teorías que aportaron un gran valor empírico, frente a los anteriores estudios de la Escuela Francesa $^{14}$. Este conjunto de obras científicas tenía como objeto el estudio de la criminalidad, en la mayoría de los casos, a través del estudio de jóvenes de las zonas más pobres de la ciudad, así como a través de las relaciones espaciales y temporales para analizar el delito. Una Escuela cuyos autores más destacados que podemos encontrar en manuales y libros de Criminología han sido en su inmensa mayoría investigadores: Park, Shaw, Mckay, Mckenzie o Burguess ${ }^{15}$. Siendo en este punto en el que debemos recordar a investigadoras que también vivieron, trabajaron e investigaron durante este periodo en la ciudad de Chicago, analizando problemas de gran interés criminológico, como son la inmigración o la prisión. Estas cuestiones que también se analizaron en esta Escuela, como podremos destacar en los siguientes apartados fueron llevadas a cabo por figuras representativas de esta corriente de pensamiento sociológico junto a Frances Alice Kellor como: Annie Marton Maclean; Sophonisba Beckinridge; Helen Thompson Woolley o Eddith Abbott $^{16}$.

Afortudamente, la Escuela de Chicago continúa viva gracias a las mujeres y hombres que dedicaron su vida a una labor científica y social. Tras un periodo de opacidad, disciplinas como la Criminología Ambiental ${ }^{17}$ y el uso de herramientas tecnológicas, permiten realizar nuevos análisis sobre el delito desde la ecología, permitido que surjan importantes investigadoras en nuestro país como Laura Vozmediano y Lucia

${ }^{11}$ La Escuela de Chicago es la cuna de la moderna Sociología Americana. Vid: García - Pablos de Molina A. (2016). Criminología: Una Introducción a sus Fundamentos Teóricos. Editorial Tirant lo Blanch. Valencia, 450 y ss. y Larrauri Pijoan, E. (2018). Introducción a la Criminología y al Sistema Penal. Madrid. Editorial Trotta.

${ }^{12}$ Algunos de los trabajos más destacados en Criminología durante este periodo, son los llevados a cabo por un grupo sociólogos, entre los que destacan Park o Burguess. Puede verse: Burguess, E.W. (1925): The City. University Chicago. Press y Shaw, C. R. Y H.D. McKay (1969). Juvenile delinquency and urban areas. A study of rates of delincuency in relation in differential characteristics of local communities in American cities, ed. Revisada. Chicago y London. The University of Chicago Press.

${ }^{13}$ Una ciudad con un enorme desarrollo industrial y económico, con una llegada de un gran número de inmigrantes procedentes de familias de antiguos esclavos y esclavas o de países de Europa, que se hacinaban en barrios con malas condiciones higiénicas y de seguridad. Un ambiente de pobreza, miseria y marginalidad, unido a dificultades para comprender el idioma y adaptarse a las costumbres de su nuevo país. Vid. Hassemer W., Muñoz Conde F. (2012). Introducción a la Criminología y a la Política Criminal. Editorial Tirant lo Blanch. Valencia.

${ }^{14}$ Guerry, A.M. (1833). Essai sur la stadistique morale de la France. París. Crochard; Quetelet, M.A. (1842). A treatise on Man. Edimburgo. Chambers.

${ }^{15}$ Investigadores de la Escuela de Chicago, con gran repercusión criminológica. Robert E. Park acuño el término “ecología del delito".

${ }^{16}$ García Dauder, S. (2010). La historia olvidada de las mujeres olvidadas de la Escuela de Chicago. Revista Española de Investigaciones Sociológicas (Reis). N ${ }^{0}$ 131, 2010.pp. 11-41.

${ }^{17}$ Vozmediano Sánz, L. y San Juan Guillen, C. (2010). Criminología Ambiental: Ecología del Delito y de la Seguridad. Editorial UOC (Barcelona) 47-52. 
Summers, que aportan, desde la criminología ambiental, estudios que comienzan a llenar nuestros manuales de Criminología ${ }^{18}$.

\section{HULL HOUSE: Un centro social y comunitario}

Un papel determinante en la figura de Frances Kellor es el centro Hull House. Una institución que fue clave para la creación de un espacio de discusión científica para muchas mujeres en la ciudad de Chicago. Una residencia que nació en uno de los barrios más deprimidos de la ciudad norteamericana, de la mano de la investigadora Jane Addams que, durante un viaje por Inglaterra acompañada de su amiga Ellen Gates Starr, visitaron el centro Toynbee Hall (1884) y les sirvió de inspiración para fundar Hull House (1889) ${ }^{19}$. Un centro social que se convirtió en un hogar para madres trabajadoras (con guardería y jardín de infancia) en un primer momento, pero que, con el paso del tiempo, también ejerció de promotor de derechos, garantías y fomentó el debate político, económico y cultural. Una institución pro-feminista, dirigida por mujeres, que ayudó a muchas mujeres a su formación. El centro social fue uno de los primeros espacios en el que se realizaron cursos universitarios y de preparación de clases. El centro Hull House se convirtió en un laboratorio para la investigación sociológica y la discusión, con la llegada al Centro de Florence Kelley. Un ejemplo de esa labor científica es la obra Hull House Maps and Papers (1895), que recoge estudios sobre inmigración, pobreza o explotación obrera, entre otras cuestiones ${ }^{20}$.

En la actualidad, el centro Hull House permanece abierto como museo, transformado en un edificio de interés en la ciudad de Chicago, es desde 1960 un monumento histórico nacional de los Estados Unidos, ubicado dentro de la Universidad de Illinois en Chicago.

\section{UNA APROXIMACIÓN A SU OBRA}

Frances Alice Kellor nació en 1873 en Columbus, Ohio. Su infancia no fue fácil, abandonada por su padre, tuvo una juventud complicada, compaginando estudios con trabajos para ayudar a su familia. A los diecisiete años comenzó a trabajar en el periódico local, donde se convirtió en periodista de investigación. A los veintidós años conoció a dos hermanas bibliotecarias (Mary y Frances Eddy) que le apoyaron y ayudaron económicamente para continuar con sus estudios.

Gracias al apoyo de sus dos compañeras de piso, Kellor se matriculó en la Facultad de Derecho de Cornell (1895), época en la que comienza a utilizar su nombre "Frances". En 1897, tras obtener el título de Derecho, se mudó a Chicago, donde le concedieron una beca para estudiar sociología en la Universidad de Chicago. En este periodo compagina sus estudios universitarios con su trabajo en un gimnasio, de donde encuentra su inspiración para realizar su primer artículo académico sobre la educación física de las mujeres y la igualdad con el hombre. Es en Chicago donde también comienza a trabajar en la Hull House.

Una de sus primeras investigaciones en la Universidad de Chicago fue en instituciones penitenciarias. Kellor analizó el tratamiento de prisiones, en particular presas negras. Esta investigación permitió la

${ }^{18}$ Summers Rodríguez, L. (2009). Las técnicas de prevención situacional del delito aplicadas a la delincuencia juvenil.

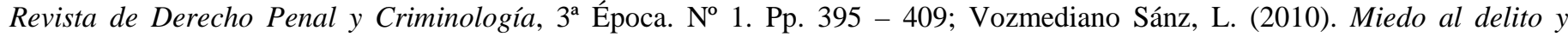
Sostenibilidad Urbana. Análisis ecológico, propuestas de medición y transferencia de resultados. Tesis Doctoral. Universidad del País Vasco.

19 Sarasa, D. (05/09/2013). “Nuevos” Centros de innovación, the Hull House, Chicago 1889. Disponible en: https://openyourcity.com/2013/05/centros-de-innovacion-urbana-the-hull-house/. [última consulta 05/11/2019].

${ }^{20}$ Adams, J. (1895). Hull-House Maps and Papers: A Presentation of Nationalities and Wages in a Congested District of Chicago, Together with Comments and Essays on Problems Growing Out of the Social Conditions. T.Y. Crowell. New York. 
publicación de su primer libro Sociología Experimental Descriptivo y Analítico: Delincuentes. Durante este periodo, es donde podemos encontrar el lado más criminológico de Frances Kellor.

Kellor se traslada a Nueva York, donde trabaja en la Oficina de Industria e Inmigración, donde comienza un estudio sobre la falta de trabajo. Una investigación con un gran trabajo de campo (Kellor, se hizo pasar como empleada para realizar el estudio). En algunas de esas investigaciones afirmaba que las agencias de empleo no eran lo que decían ser (no tenían un lugar físico o no realizaban ninguna selección de las personas trabajadoras o), mostrando a su vez su preocupación por la inmigración y las mujeres negras; lo que la llevó a identificar nuevos sistemas de esclavitud en el trabajo. Esta lucha contra el trato a la población inmigrante a la hora de ofrecer trabajos dignos se refleja en el libro Out of Work (1904). Su trabajo sobre las agencias de empleo le permitió conocer a Mary Dreier y, juntas promovieron la aprobación de normas que frenaron los abusos de las agencias de empleo sobre personas inmigrantes en la ciudad de New York, y crearon el Comité Intermunicipal de Investigación de Hogares. Un aspecto importante en la vida de Kellor, de gran interés criminológico, es, sin duda, su labor en una organización que busca erradicar la prostitución. En 1906 fundó la Liga Nacional para la Protección de las Mujeres de Color.

Durante este periodo llevó a cabo un proyecto de investigación sumamente amplio sobre el tratamiento de inmigrantes en New York, Boston, Filadelfia y Chicago, que sirvió al presidente de los Estados Unidos, Theodore Roosevelt, para incorporar sus conclusiones a sus políticas sobre inmigración. Estas investigaciones en materia de inmigración le llevaron a su vez a solicitar la creación de una Oficina de Industrias e Inmigración, cuya creación permitió proporcionar arbitraje e información a inmigrantes para evitar posibles explotaciones.

Su vida política comenzó en 1912 a través de su participación en el partido progresista, donde pudo poner en marcha, muchas de las ideas planteadas en su faceta investigadora, como programas de asimilación para inmigrantes (clases de inglés, lecciones de ciudadanía, etc.)

La Primera Guerra Mundial hizo cambiar las prioridades de Kellor en su defensa de los inmigrantes, centrándose en su asimilación. En 1916 escribió Straight America: A call to National Service ${ }^{21}$, y cuatro años más tarde publicó su obra Immigration and the Future ${ }^{22}$, donde continúa con su defensa sobre la acogida de la población inmigrante.

En 1931, elaboró el Código de Arbitraje, dentro de su labor en la Oficina de la Asociación Americana de Arbitraje. Su trabajo fue reconocido a nivel mundial. Falleció en New York, el 4 de enero de $1952^{23}$.

\section{CONSIDERACIONES FINALES}

La Escuela de Chicago fue precursora de las teorías ecológicas más estudiadas en Criminología, pero también nos brindó estudios e investigaciones sobre otros temas, que resultan de gran interés científico y académico. Investigaciones realizadas por científicas que quedaron relegadas a un segundo plano entre el conjunto de numerosas teorías criminológicas que hemos podido estudiar. Hoy, ninguna persona puede obviar el interés criminológico de la inmigración ${ }^{24}$ o las prisiones ${ }^{25}$, ni su importancia en la configuración de

\footnotetext{
${ }^{21}$ Kellor, F. (1916). Straight America: A call to National Service. Editorial Macmillan.

${ }^{22}$ Kellor, F. Immigration and the future. Creative Media Partners, LLC, 2019.
}

\footnotetext{
${ }^{23}$ Kenneth Press, J. (2012). Founding Mother: Frances Kellor and the Creation of Modern America.

${ }^{24}$ García España, E. (2001). Inmigración y Delincuencia en España: Análisis criminológico. Editorial Tirant lo Blanch $1^{\circ}$ Edición. Valencia.

${ }^{25}$ Caravaca Sánchez, F., Sánchez Rodríguez, F. y Luna Maldonado, F. (2013). La situación de las mujeres en las prisiones de Murcia. ¿Más vulnerables que los hombres? Boletín Criminológico. N ${ }^{a}$ 146; Cuaresma Morales, D. y Nicolás
} 
la realidad social actual. El discurso político que escuchamos en nuestro país en estos últimos meses por parte de algunos partidos políticos resulta similar al que estamos escuchando en estos últimos años en otros países de Europa (a modo de ejemplo, los gobiernos italiano y húngaro), y pone de manifiesto la importancia de realizar investigaciones y políticas en este sentido. Un mensaje xenófobo y racista que estigmatiza a este colectivo, relacionándolo con delincuencia y terrorismo, y que desde el ámbito académico debemos combatir $^{26}$. El trabajo de Frances Kellor es un ejemplo de orientación del ámbito académico hacia la lucha para desmontar el discurso de odio al que estamos asistiendo, planteando como alternativa una política de integración y asimilación, políticas, en mi opinión, tan importantes o más, que las políticas diseñadas para responder a la llegada del inmigrante, y que debería ser inspirador para el desarrollo de la Criminología en nuestro país.

Por otro lado, también podemos seguir su ejemplo en el ámbito penitenciario, siendo la cárcel una de las instituciones que con más interés analiza la Criminología, y que acompaña al complejo e importante ámbito de la reinserción y rehabilitación de los presos. Frances Kellor, no sólo realizó estudios al respecto, hemos podido comprobar que también aplicó las políticas que previamente había estudiado ${ }^{27}$. En pocas palabras, aplicó las políticas públicas bajo criterios científicos refutados. Hecho que constantemente buscamos en Criminología, desarrollando vías para su aplicación en Políticas Criminales.

En definitiva, Frances Alice Kellor fue una criminóloga en un mundo de hombres, y recuperar su figura nos ayuda a rescatar investigaciones de gran interés criminológico, así como destacar el papel de la mujer en la Escuela de Chicago.

\section{AGRADECIMIENTOS}

Los autores quieren agradecer de manera especial, la labor investigadora de la profesora Silvia García Dauder de la Universidad Rey Juan Carlos, por descubrirnos a una criminóloga dentro de la Escuela de Chicago, que los manuales de criminología han obviado durante años.

\section{BIBLIOGRAFÍA}

- $\quad$ ADAMS, J. (1895). Hull-House Maps and Papers: A Presentation of Nationalities and Wages in a Congested District of Chicago, Together with Comments and Essays on Problems Growing Out of the Social Conditions. T.Y. Crowell. New York.

- $\quad$ ADLER, F. (1975). SISTER IN CRIME. THE RISE OF THE NEW FEMALE CRIMINAL. NY. MC-GRAW-HILL.

- $\quad$ BURGUESS, E.W. (1925): The City. University Chicago. Press.

- $\quad$ CARAVACA SÁNCHEZ, F., SÁNCHEZ RODRÍGUEZ, F. Y LUNA MALDONADO, F. (2013). La situación de las mujeres en las prisiones de Murcia. ¿Más vulnerables que los hombres? Boletín Criminológico. Na 146.

Soriano, L. (2013). Mujeres a la sombra: Influencia del género en las actitudes profesionales de los funcionarios penitenciarios. Revista Española De Investigación Criminológica, 11, 1-29

${ }^{26}$ García España, E. (2018). Enfoques criminológicos de las migraciones, de Elisa García España, Síntesis. Madrid.

${ }^{27}$ Nguyen, T., Frerich, N., Redondo Illescas, S. y Andrés - Pueyo, A. (2014). Reinserción y gestión del riesgo de reincidencia en agresores sexuales excarcelados: El proyecto “círculos de apoyo y responsabilidad” en Cataluña. Boletín Criminológico. № 151. 
- $\quad$ CUARESMA MORALES, D. Y NICOLÁS SORIANO, L. (2013). Mujeres a la sombra: Influencia del género en las actitudes profesionales de los funcionarios penitenciarios. Revista Española De Investigación Criminológica, 11, 1-29.

- GARCÍA DAUDER, S. (2010). La historia olvidada de las mujeres olvidadas de la Escuela de Chicago. Revista Española de Investigaciones Sociológicas (Reis). No 131, 2010.pp. 11-41.

- GARCÍA-PABLOS DE MOLINA, A. (2016). Criminología: Una Introducción a sus Fundamentos Teóricos. Editorial Tirant lo Blanch. Valencia, 450 y ss.

- GARCÍA ESPAÑA, E. (2001). Inmigración y Delincuencia en España: Análisis criminológico. Editorial Tirant lo Blanch $1^{\circ}$ Edición. Valencia.

- $\quad$ GARCÍA ESPAÑA, E. (2018). Enfoques criminológicos de las migraciones, de Elisa García España, Síntesis. Madrid.

- $\quad$ GUERRY, A.M. (1833). Essai sur la stadistique morale de la France. París. Crochard.

- $\quad$ HASSEMER W., MUÑOZ CONDE F. (2012). Introducción a la Criminología y a la Política Criminal. Editorial Tirant lo Blanch. Valencia.

- $\quad$ KELLOR, F. (1916). Straight America: A call to National Service. Editorial Macmillan.

- $\quad$ KELLOR, F. Immigration and the future. Creative Media Partners, LLC, 2019.

- $\quad$ KENNETH PRESS, J. (2012). Founding Mother: Frances Kellor and the Creation of Modern America.

- $\quad$ LARRAURI PIJOAN, E. (2018). Introducción a la Criminología y al Sistema Penal. Madrid. Editorial Trotta.

- $\quad$ NGUYEN, T., FRERICH, N., REDONDO ILLESCAS, S. Y ANDRÉS - PUEYO, A. (2014). Reinserción y gestión del riesgo de reincidencia en agresores sexuales excarcelados: El proyecto “círculos de apoyo y responsabilidad” en Cataluña. Boletín Criminológico. № 151.

- $\quad$ QUETELET, M.A. (1842). A treatise on Man. Edimburgo. Chambers.

- SALAZAR BENÍTEZ, O. (2018). El hombre que no deberíamos ser (1ª Edición) Barcelona. Editorial Planeta.

- SARASA, D. (05/09/2013). “Nuevos” Centros de innovación, the Hull House, Chicago 1889. Disponible en: https://openyourcity.com/2013/05/centros-de-innovacion-urbana-the-hull-house/. [última consulta 05/11/2019].

- SERRANO GÓMEZ, A. (2007). Historia de la Criminología en España. Madrid. Dykinson.

- SERRANO TÁRRAGA Ma D. (2014). El Rol de la Criminología para la Seguridad en la Sociedad Contemporánea. Madrid Dykinson 233-234.

- SHAW, C. R. Y MCKAY, H.D. (1969). Juvenile delinquency and urban areas. A study of rates of delincuency in relation in differential characteristics of local communities in American cities, ed. Revisada. Chicago y London. The University of Chicago Press.

- SIMON, R.J. (1975). Women and crime. Lexington, M.A: D.C. Health.

- SUMMERS RODRÍGUEZ, L. (2009). Las técnicas de prevención situacional del delito aplicadas a la delincuencia

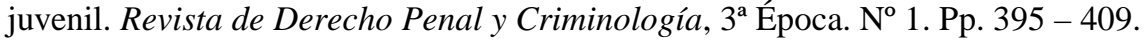

- TORRES DÍAZ, M.C. (2018). Dret i Criminología: Guies per una docència universitaria amb perspectiva de génere. Xarxa Vives d'universitat. Disponible en: http://www.vives.org/serveis/guies-per-a-una-docencia-universitaria-ambperspectiva-de-genere/dret-i-criminologia/.

- VOZMEDiAnO SÁNZ, L. Y SAN JUAN GUILLEN, C. (2010) Criminología Ambiental: Ecología del Delito y de la Seguridad. Editorial UOC (Barcelona) 47-52.

- VOZMEDIANO SÁNZ, L. (2010). Miedo al delito y Sostenibilidad Urbana. Análisis ecológico, propuestas de medición y transferencia de resultados. Tesis Doctoral. Universidad del País Vasco.

- $\quad$ WALKER, S. y BARTON, L. (1983). Gender, class and education. Nueva York: The Falmer Press. 